\title{
Distributed Attitude Consensus for Multiple Rigid Spacecraft under Jointly Connected Switching Topologies
}

Long Ma, Shicheng Wang, Haibo Min, Shouyi Liao, and Zhiguo Liu

High-Tech Institute of Xian, Shaanxi 710025, China

Correspondence should be addressed to Long Ma; mlong_301@163.com

Received 4 November 2015; Revised 16 March 2016; Accepted 29 March 2016

Academic Editor: Yang Shi

Copyright @ 2016 Long Ma et al. This is an open access article distributed under the Creative Commons Attribution License, which permits unrestricted use, distribution, and reproduction in any medium, provided the original work is properly cited.

\begin{abstract}
We study the distributed leader-following attitude consensus problem for multiple rigid spacecraft with a single leader under jointly connected switching topologies. Two cases are considered, where the first case is with a static leader and the second case is with a dynamic leader. By constructing an auxiliary vector and a distributed observer for each follower spacecraft, the controllers are designed to drive all the attitudes of the follower spacecraft to the leader's, respectively, for both of the two cases, though there are some time intervals in which the communication topology is not connected. The whole system is proved to be stable by using common Lyapunov function method. Finally, the theoretical result is illustrated by numerical simulations.
\end{abstract}

\section{Introduction}

In recent years, spacecraft formation flying (SFF) has become a new technology that plays an important role in the future space missions, such as Earth Observing (EO) [1, 2], Orbit Express (OE), Terrestrial Planet Finder (TPF) [3], Space Telescope Assembly (STA), Stellar Imager (SI), and SyntheticAperture Imaging (SAI) [4]. This interest is motivated by the advantages gained by replacing a traditionally large and expensive spacecraft by a cluster of microspacecraft to accomplish a common task in a coordinated manner [5]. One of the most important control goals for SFF is the attitude consensus or alignment, where every spacecraft updates its own orientation using the orientations of its local neighbors. As a result, the orientations of all spacecrafts approach a common value. For example, in interferometry applications, it is often essential to control different spacecraft to maintain the same or relative attitudes during and after formation manoeuvres. Since the angular velocity of the body cannot be integrated to obtain the attitude of the body directly because of the nonlinear dynamics [6], attitude coordination control problem becomes a particularly interesting problem for the researchers. Reference [7] solves the synchronized multiple spacecraft rotations control problem with a passivity-based damping method, while [8] focuses on the condition that the angular velocity is unknown. Other interesting problems include attitude consensus with time delay [9], with input constraints [10], with attitude constraints [11], and with multiple leaders [12]. Certainly, it will be more challenging if only a subset of group agents have access to the virtual leader [13].

As we all know, the biggest difference between multiagent systems and single-agent system lies in the communication network. Therefore, the characteristics of networks decide the performance of the whole system to a great extent. Communication outage, new member's joining or quitting, radio silence, or recovery will change the communication topology (termed as switching topology), which makes it more difficult to design the control laws. Based on relative attitude information and Modified Rodriguez Parameters (MRPs), [14] considers cooperative attitude tracking problem and gives a control law in the presence of a dynamic communication topology. Reference [15] extends this to the condition that there exist both multiple time-varying communication delays and dynamically changing topologies, and the result of uniformly ultimate boundedness of the closed-loop system is obtained. Considering more complicating elements, [16] presents controllers that can render 
a spacecraft formation consistent to a given trajectory globally with dynamic information exchange graph and nonuniform time-varying delays while coping with the parameter uncertainties and unexpected disturbances. In [17], a 6DOF dynamics model of the spacecraft formation flying is established in Euler-Lagrange form, and a control algorithm based on consensus theory is proposed in the presence of dynamic communication topology. Furthermore, almostglobal attitude synchronization is achieved in [18] based on switching joint connection; however auxiliary variables are introduced which make the controllers complicated. In [19], by utilizing Lyapunov direct method and choosing a common Lyapunov function properly, the robustness of the designed position and attitude coordinated controllers to communication delays, switching topologies, parameter uncertainties, and external disturbances is guaranteed.

Note that all the above literatures only consider the uniformly connected topologies. However, the jointly connected case is more challenging because there will be isolated agents during some time intervals, which makes the controller designing more challenging and more difficult. It is worth noting that [20] addresses the attitude synchronization problem of multiple rigid body agents in $\mathrm{SO}(3)$ with directed and jointly strongly connected interconnection topologies. Using the axis-angle representation of the orientation, a distributed controller is proposed based on relative orientations between the agents without a global reference coordinate frame. And from the viewpoint of interior metrics, [21] provides a leaderless consensus protocol for strongly convex geodesic balls and applies it to the consensus problem of rotation attitudes under switching and directed communication topologies. Although the topologies are jointly connected, there is no leader in the system, and the control schemes are not used in the leaderfollowing problem and especially not used in the dynamic tracking problem.

In this paper, we focus on the leader-following attitude consensus problem under jointly connected topologies, where the attitude of the leader is only available to a subset of the followers. The difficulty lies in how to construct an effective controller that can drive all the attitudes of the followers not only to a same constant value but also to the attitude of the dynamic leader. We use MRPs to represent the spacecraft attitude for nonredundancy. By constructing a useful auxiliary vector for each spacecraft, we design a distributed controller to each follower to guarantee that the attitude errors between the followers and the static leader converge to zero. By associating a distributed observer for each follower, the controller is designed such that the attitude of the followers will converge to the dynamic leader.

The remainder of this paper is organized as follows. In Section 2, we present the dynamics of rigid body attitude, basic knowledge of graph theory, and the statement of leader-following attitude consensus problem under jointly connected topologies. The details about the construction of auxiliary vectors and distributed observers as well as derivation of the controller are presented in Section 3. In Section 4, we show simulation results for five spacecrafts using the control laws proposed in Section 3 and conclusion follows in Section 5.
Notation 1. $I_{n}$ denotes the $n$-order unit matrix. One has $\mathbb{R}:=$ $(-\infty, \infty), \mathbb{R}_{>0}:=(0, \infty), \mathbb{R}_{\geq 0}:=[0, \infty) . \lambda_{m}\{\mathbf{A}\}$ and $\lambda_{M}\{\mathbf{A}\}$ represent the minimum and maximum eigenvalues of matrix $\mathbf{A}$, respectively. $\|\mathbf{A}\|_{2}$ is the spectral norm of matrix $\mathbf{A} .|\mathbf{x}|$ stands for the standard Euclidean norm of the vector $\mathbf{x} \in$ $\mathbb{R}^{n}$. For any function $\mathbf{f}: \mathbb{R}_{\geq 0} \rightarrow \mathbb{R}^{n}$, the $\mathscr{L}_{\infty}$-norm is defined as $\|\mathbf{f}\|_{\infty}=\sup _{t \geq 0}|\mathbf{f}(t)|$, and the $\mathscr{L}_{2}$-norm as $\|\mathbf{f}\|_{2}^{2}=$ $\int_{0}^{\infty}|\mathbf{f}(t)|^{2} d t$. The $\mathscr{L}_{\infty}$ and $\mathscr{L}_{2}$ spaces are defined as the sets $\left\{\mathbf{f}: \mathbb{R}_{\geq 0} \rightarrow \mathbb{R}^{n}:\|\mathbf{f}\|_{\infty}<\infty\right\}$ and $\left\{\mathbf{f}: \mathbb{R}_{\geq 0} \rightarrow \mathbb{R}^{n}:\|\mathbf{f}\|_{2}<\right.$ $\infty$ \}, respectively. $\varrho(t)$ is used to denote a piecewise constant switching signal $\varrho:[0,+\infty) \rightarrow \mathscr{P}=1,2, \ldots, \rho$ for some positive integer $\rho$, where $\mathscr{P}$ is called the switching index set. We assume that the switching instants $t_{0}=0, t_{1}, t_{2}, \ldots$ of $\varrho$ satisfy $t_{k+1}-t_{k} \geq \tau>0$ for some constant $\tau$ and any $k \geq 0$, and $\tau$ is called the dwell time.

\section{Problem Statement and Background Information}

2.1. Dynamics of Rigid Spacecraft. Consider a multiple rigid spacecraft system consisting of $n+1$ spacecraft. Suppose that there exist $n$ followers, labeled as agents 1 to $n$, and one single leader labeled as agent 0 . The dynamics of the $n$ followers are given by

$$
\begin{aligned}
& \dot{\sigma}_{i}=G\left(\sigma_{i}\right) \omega_{i}, \\
& \dot{\omega}_{i}=J_{i}^{-1}\left(-\omega_{i}^{\times} J_{i} \omega_{i}+u_{i}\right),
\end{aligned}
$$

where $\sigma_{i} \in \mathbb{R}^{3}$ denotes the Modified Rodrigues Parameters that represent the attitude of the $i$ th spacecraft. Here $\sigma_{i}$ is defined by $\sigma_{i}=\widehat{e}_{i} \tan \left(\phi_{i} / 4\right)$, where $\hat{e}_{i}$ is the Euler axis and $\phi_{i}$ is the Euler angle [22]. $\omega_{i}=\left[\begin{array}{lll}\omega_{i 1} & \omega_{i 2} & \omega_{i 3}\end{array}\right]^{T} \in \mathbb{R}^{3}$ denotes the angular velocity of the $i$ th spacecraft; $J_{i}$ and $u_{i}$ are, respectively, the inertial matrix and the external input torque of the $i$ th spacecraft. $\omega_{i}^{\times}$is the skew-symmetric matrix with the form

$$
\omega_{i}^{\times}=\left[\begin{array}{ccc}
0 & -\omega_{i 3} & \omega_{i 2} \\
\omega_{i 3} & 0 & -\omega_{i 1} \\
-\omega_{i 2} & \omega_{i 1} & 0
\end{array}\right] .
$$

The matrix $G\left(\sigma_{i}\right)$ is given by

$$
G\left(\sigma_{i}\right)=\frac{1}{2}\left[\frac{\left(1-\sigma_{i}^{T} \sigma_{i}\right) I_{3}}{2}+\sigma_{i}^{\times}+\sigma_{i} \sigma_{i}^{T}\right],
$$

which has the following properties $[23,24]$ :

$$
\begin{aligned}
\sigma_{i}^{T} G\left(\sigma_{i}\right) \omega_{i} & =\frac{1+\sigma_{i}^{T} \sigma_{i}}{4} \sigma_{i}^{T} \omega_{i}, \\
G\left(\sigma_{i}\right) G^{T}\left(\sigma_{i}\right) & =\left(\frac{1+\sigma_{i}^{T} \sigma_{i}}{4}\right)^{2} I_{3}=p_{i} I_{3} .
\end{aligned}
$$

Remark 1. We hasten to point out that the use of the MRPs simplifies the analysis and the ensuing formulas, since there 
is no additional equality constraint to worry about. Another advantage of the MRPs is the fact that they can parameterize eigenaxis rotations up to 360 degrees. In contrast, other three-dimensional parameterizations are limited to eigenaxis rotations of less than 180 degrees. One can refer to $[22,25]$ for more details. The stability results obtained in this paper mean the stability of the corresponding kinematic parameters. That is, the stability is guaranteed for all initial attitudes except for the singular point $\Phi_{i}= \pm 360^{\circ}$, where $\Phi_{i}$ is the principle angle of the attitude of the $i$ th rigid body.

2.2. Graph Theory. Graphs can be conveniently used to represent the information flow between agents. Let $\mathscr{G}=$ $\{\mathscr{V}, \mathscr{E}, \mathscr{A}\}$ be an undirected graph or directed graph (digraph) of order $n$ with the set of nodes $\mathscr{V}(\mathscr{G})=\left\{v_{1}, v_{2}, \ldots, v_{n}\right\}$, the set of edges $\mathscr{E} \subseteq \mathscr{V} \times \mathscr{V}$, and a weighted adjacency matrix $\mathscr{A}=\left[a_{i j}\right]$ with nonnegative adjacency elements $a_{i j}$. The node indices belong to a finite index set $l=\{1,2, \ldots, n\}$. The set of neighbors of node $v_{i}$ is the set of all nodes which point (communicate) to $v_{i}$, denoted by $\mathscr{N}_{i}=\left\{v_{j} \in \mathscr{V}:\left(v_{i}, v_{j}\right) \in\right.$ $\varepsilon(\mathscr{G})\}$. The graph adjacency matrix $\mathscr{A}=\left[a_{i j}\right], \mathscr{A} \in \mathbb{R}^{n \times n}$, is such that $a_{i j}>0$ if $j \in \mathcal{N}_{i}$ and $a_{i j}=0$ otherwise. $\mathscr{D}=\operatorname{diag}\left[d_{1}, \ldots, d_{n}\right] \in \mathbb{R}^{n \times n}$ is called the degree matrix of $\mathscr{G}$, where $d_{i}=\sum_{j=1}^{n} a_{i j}$. The weighted Laplacian matrix of $\mathscr{G}$ is $\mathscr{L}=\mathscr{D}-\mathscr{A}$. A digraph $\mathscr{G}_{s}=\left\{\mathscr{V}_{s}, \mathscr{E}_{s}\right\}$ is a subgraph of $\mathscr{G}=\{\mathscr{V}, \mathscr{E}\}$ if $\mathscr{V}_{s} \subseteq \mathscr{V}$ and $\mathscr{E}_{s} \subseteq \mathscr{E} \cap\left(\mathscr{V}_{s} \times \mathscr{V}_{s}\right)$. Given a set of $r$ digraphs $\left\{\mathscr{G}_{i}=\left(\mathscr{V}, \mathscr{E}_{i}\right), i=1, \ldots, r\right\}$, the digraph $\mathscr{G}=(\mathscr{V}, \mathscr{E})$ where $\mathscr{E}=\bigcup_{i=1}^{r} \mathscr{E}_{i}$ is called the union of digraphs $\mathscr{G}_{i}$, denoted by $\mathscr{G}=\bigcup_{i=1}^{r} \mathscr{G}_{i}$.

Given a piecewise constant switching signal $\varrho(t)$, we can define a nonnegative switching matrix $\overline{\mathscr{A}}_{\rho(t)}=\left[a_{i j}(t)\right], i, j=$ $0,1, \ldots, n$, where, for $i=1, \ldots, n, a_{i 0}(t)>0$ if and only if the control input $u_{i}$ can access the information of the leader at time instant $t$, and all other elements of $\overline{\mathscr{A}}_{\rho(t)}$ are arbitrary nonnegative numbers satisfying $a_{i i}(t)=0$ for any $t \geq 0, i=0,1, \ldots, n$. Let $\overline{\mathscr{G}}_{\varrho(t)}=\left(\overline{\mathscr{V}}, \overline{\mathscr{E}}_{\varrho(t)}\right)$ be a dynamic digraph of $\overline{\mathscr{A}}_{\varrho(t)}$. Then, the node set $\overline{\mathscr{V}}=0,1, \ldots, n$ with 0 corresponding to the leader system and the integer $i, i=$ $1, \ldots, n$, corresponding to the $i$ th subsystem of the follower system, and $\overline{\mathscr{E}}_{\varrho(t)} \subseteq \overline{\mathscr{V}} \times \overline{\mathscr{V}}$ and $(i, j) \in \overline{\mathscr{E}}_{\varrho(t)}$ if and only if $a_{i j}(t)>0$ at time instant $t$.

To model the jointly connected topologies, we consider an infinite sequence of continuous, bounded, nonoverlapping time intervals $\left[t_{k}, t_{k+1}\right), k=0,1,2, \ldots$ with $t_{0}=0, T_{0} \leq$ $t_{k+1}-t_{k} \leq T$ for some constants $T_{0}$ and $T$. Assume that each interval $\left[t_{k}, t_{k+1}\right)$ is composed of the following nonoverlapping subintervals $\left[t_{k}^{0}, t_{k}^{1}\right), \ldots,\left[t_{k}^{j-1}, t_{k}^{j}\right), \ldots,\left[t_{k}^{m_{k}-1}, t_{k}^{m_{k}}\right)$ with $t_{k}^{0}=t_{k}$ and $t_{k}^{m_{k}}=t_{k+1}$ for some nonnegative integer $m_{k}$. The topology switches at time instants $t_{k}^{0}, t_{k}^{1}, \ldots, t_{k}^{m_{k}-1}$, which satisfy $t_{k}^{j-1}-t_{k}^{j} \geq \tau, j=1, \ldots, m_{k}$, with $\tau$ a positive constant, such that, during each subinterval $\left[t_{k}^{j-1}, t_{k}^{j}\right)$, the interconnection topology $\mathscr{G}_{\rho(t)}$ does not change. Note that, in each interval $\left[t_{k}, t_{k+1}\right), \mathscr{G}_{\rho(t)}$ is permitted to be disconnected. The graphs are said to be jointly connected across the time interval $[t, t+T], T>0$ if the union of graphs $\mathscr{G}_{\rho(t)}: s \in$ $[t, t+T]$ is connected [26].
2.3. Problem Statement. In this paper, we consider the leaderfollowing attitude synchronization control problem under jointly connected graph with two cases, that is, regulation case and dynamic tracking case.

As for the regulation case, the leader is set to the desired attitude with no angular velocity. The control objective is to drive the attitudes of the followers to the static leader with a dynamic network topology $\overline{\mathscr{G}}_{\varrho(t)}$; that is,

$$
\begin{aligned}
\left|\sigma_{i}(t)-\sigma_{0}\right| & \longrightarrow 0, \\
\left|\omega_{i}(t)\right| \longrightarrow 0, & \\
& i=1, \ldots, n .
\end{aligned}
$$

As for the dynamic tracking case, the leader is set to the desired attitude with nonzero angular velocity. The control objective is to drive the attitudes and the angular velocities of the followers to the dynamic leader with a dynamic network topology $\overline{\mathscr{G}}_{\varrho(t)}$; that is,

$$
\begin{aligned}
\left|\sigma_{i}(t)-\sigma_{0}(t)\right| & \longrightarrow 0, \\
\left|\dot{\sigma}_{i}(t)-\dot{\sigma}_{0}(t)\right| & \longrightarrow 0, \\
i & =1, \ldots, n .
\end{aligned}
$$

Assumption 2. The communication among the followers is bidirectional, and there exists a subsequence $\left\{i_{k}\right\}$ of $\{i: i=$ $0,1, \ldots\}$ with $t_{i_{k+1}}-t_{i_{k}}<T$ for some positive $T$ such that the union graph $\bigcup_{j=i_{k}}^{i_{k+1}-1} \frac{i_{k}}{\mathscr{G}_{\varrho}}\left(t_{j}\right)$ contains a spanning tree with the node 0 as the root.

Lemma 3 (see [27]). Function $x(t):[0,+\infty) \rightarrow \mathbb{R}$ is continuously differentiable, and $\lim _{t \rightarrow \infty} x(t)$ exists. If $\ddot{x}(t)$ exists and $\ddot{x}(t)$ is bounded, then $\lim _{t \rightarrow \infty} \dot{x}(t)=0$.

Lemma 4 (see [28]). Let $t_{i}, i=0,1,2, \ldots$ be a sequence satisfying $t_{0}=0, t_{i+1}-t_{i} \geq \tau>0$. Suppose that a scalar continuous function $V(t):[0,+\infty) \rightarrow \mathbb{R}$ satisfies the following:

(1) $V(t)$ is lower bounded.

(2) $\dot{V}(t)$ is differentiable and nonpositive on each interval $\left[t_{i}, t_{i+1}\right)$.

(3) $\ddot{V}(t)$ is bounded over $[0,+\infty)$ in the sense that there exists a positive constant $\xi$ such that

$$
\sup _{t_{i} \leq t \leq t_{i+1},}|\ddot{V}(t)| \leq \xi, 1,2, \ldots .
$$

Then $\dot{V}(t) \rightarrow 0$ as $t \rightarrow+\infty$.

\section{Main Results}

In this section, we deal with the leader-following attitude consensus problem with jointly connected topologies. Firstly, we associate each agent with the following auxiliary variable vector:

$$
s_{i}=\dot{\sigma}_{i}+c \sigma_{i}
$$


where $c$ is a positive constant. According to (9), we get $s_{0}=$ $\dot{\sigma}_{0}+c \sigma_{0}$ for the leader.

3.1. Regulation Case. In order to reflect the isolated agents for jointly connected topologies at some instants, we denote $l_{c}$ as the set of the connected agents except for the leader, and $l_{s}$ as the set of the isolated agents at time instant $t$, respectively. Obviously, we have $l_{c} \cup l_{s}=l$ and $l_{c} \cap l_{s}=\varnothing$. The control law for the $i$ th follower is designed as

$$
u_{i}=\omega_{i}^{\times} J_{i} \omega_{i}+\frac{J_{i} G^{T}\left(\sigma_{i}\right)}{p_{i}}\left[z_{i}-\gamma \sum_{j \in \bar{N}_{i}} a_{i j}\left(s_{i}-s_{j}\right)\right] \text {, }
$$

where $\gamma>0$ is a positive constant, $z_{i}=-\dot{G}\left(\sigma_{i}\right) \omega_{i}-c \dot{\sigma}_{i}$, and $p_{i}$ is defined as in (5).

If we define

$$
s_{i 0}=s_{i}-s_{0},
$$

then we get that

$$
s_{i}-s_{j}=s_{i 0}-s_{j 0} .
$$

Combining (1) with (9)-(12), the dynamics of the connected spacecraft can be written as

$$
\begin{aligned}
& \dot{s}_{i 0}=\ddot{\sigma}_{i}+c \dot{\sigma}_{i}=\dot{G}\left(\sigma_{i}\right) \omega_{i}+G\left(\sigma_{i}\right) \dot{\omega}_{i}+c \dot{\sigma}_{i} \\
&=\dot{G}\left(\sigma_{i}\right) \omega_{i}+G\left(\sigma_{i}\right) J_{i}^{-1}\left(-\omega_{i}^{\times} J_{i} \omega_{i}+u_{i}\right)+c \dot{\sigma}_{i} \\
&=-\gamma \sum_{j \in \bar{N}_{i}} a_{i j}(t)\left(s_{i}-s_{j}\right)=-\gamma \sum_{j \in \bar{N}_{i}} a_{i j}(t)\left(s_{i 0}-s_{j 0}\right), \\
& i \in l_{c},
\end{aligned}
$$

where the last equation has used (12), and the dynamics of the isolated spacecraft can be written as

$$
\dot{s}_{i 0}=0, \quad i \in l_{s}
$$

which can be synthesized as

$$
\dot{s}_{i 0}= \begin{cases}-\gamma \sum_{j \in \bar{N}_{i}} a_{i j}(t)\left(s_{i 0}-s_{j 0}\right), & i \in l_{c}, \\ 0, & i \in l_{s} .\end{cases}
$$

Remark 5. For jointly connected topologies, there may exist connected spacecraft and isolated spacecraft at the same time in some time intervals. It is possible that some agents are not connected to the leader; however they are connected to each other. In this case, the dynamics of these agents can still be converted to (13).

From the above, we get the following result.

Theorem 6. Under Assumption 2, the leader-following consensus of system (1) is achieved by choosing the control protocols as (10).
Proof. Define a Lyapunov function candidate as

$$
V=\frac{1}{2} \sum_{i=1}^{n} V_{i}(t)
$$

where

$$
V_{i}=\frac{1}{2} s_{i 0}^{T} s_{i 0}
$$

Note that $V(t)$ is continuously differentiable in spite of the existence of the switching topologies. Then, $V(t)$ can be divided into two parts; that is, $V(t)=V_{c}(t)+V_{s}(t)$, where $V_{c}(t)=\sum_{i \in l_{c}} V_{i}(t)$ and $V_{s}(t)=\sum_{i \in l_{s}} V_{i}(t)$.

Taking the derivative of $V_{c}(t)$ gives

$$
\begin{aligned}
\dot{V}_{c}(t)= & \sum_{i \in l_{c}} s_{i 0}^{T} \dot{s}_{i 0}=-\gamma \sum_{i \in l_{c}} s_{i 0}^{T} \sum_{j \in \bar{N}_{i}} a_{i j}(t)\left(s_{i 0}-s_{j 0}\right) \\
= & -\gamma \sum_{i \in l_{c}} \sum_{j \in N_{i}} a_{i j}(t) s_{i 0}^{T}\left(s_{i 0}-s_{j 0}\right)-\gamma a_{i 0}(t) s_{i 0}^{T} s_{i 0} \\
= & -\frac{1}{2} \gamma \sum_{i \in l_{c}} \sum_{j \in N_{i}} a_{i j}(t)\left(s_{i 0}-s_{j 0}\right)^{T}\left(s_{i 0}-s_{j 0}\right) \\
& -\gamma a_{i 0}(t) s_{i 0}^{T} s_{i 0} .
\end{aligned}
$$

Here, we have used the fact that

$$
\begin{aligned}
& \sum_{i \in l_{c}} \sum_{j \in N_{i}} a_{i j}(t) s_{i 0}^{T}\left(s_{i 0}-s_{j 0}\right) \\
& \quad=\frac{1}{2} \sum_{i \in l_{c}} \sum_{j \in N_{i}} a_{i j}(t)\left(s_{i 0}-s_{j 0}\right)^{T}\left(s_{i 0}-s_{j 0}\right),
\end{aligned}
$$

which is based on the assumption that $\mathscr{G}$ is undirected.

Similarly, taking the derivative of $V_{s}(t)$ gives

$$
\dot{V}_{s}(t)=\sum_{i \in l_{s}} s_{i 0}^{T} \dot{s}_{i 0}=0 .
$$

From (18) and (20), we get that $V(t) \geq 0$ and $\dot{V}(t) \leq$ 0 , which implies that $\lim _{t \rightarrow \infty} V(t)=V(\infty)$ exists. Thus it follows that $s_{i 0}-s_{j 0} \in \mathscr{L}_{2}$ and $s_{i 0} \in \mathscr{L}_{\infty}$. Together with (15), we get that $\dot{s}_{i 0} \in \mathscr{L}_{\infty}$. As

$$
\begin{aligned}
\ddot{V}(t)= & \ddot{V}_{c}(t) \\
= & -\gamma \sum_{i \in l_{c}} \sum_{j \in N_{i}} a_{i j}(t)\left(s_{i 0}-s_{j 0}\right)^{T}\left(\dot{s}_{i 0}-\dot{s}_{j 0}\right) \\
& -2 \gamma a_{i 0}(t) s_{i 0}^{T} \dot{s}_{i 0} \leq 0,
\end{aligned}
$$

then we can conclude that $\ddot{V}(t) \in \mathscr{L}_{\infty}$.

By invoking Lemma 3, we get $\lim _{t \rightarrow \infty} \dot{V}(t)=0$. From Assumption 2, there exists a constant $K>0$ and, $\forall k \geq K$, we choose $k$ such that the time interval $\left[t_{k}, t_{k+1}\right)$ encompasses some time intervals across which the communication topologies are jointly connected. With (18), it follows that $\lim _{t \rightarrow \infty} s_{i 0}=\lim _{t \rightarrow \infty} s_{j 0}=0$, together with $s_{i 0}=\dot{\sigma}_{i 0}+\sigma_{i 0}=$ $\dot{\sigma}_{i}+\sigma_{i 0}$; we get that $\lim _{t \rightarrow \infty} \dot{\sigma}_{i 0}=-\lim _{t \rightarrow \infty} \sigma_{i 0}$. Then we get that if $\sigma_{i 0}>0$, then $\dot{\sigma}_{i 0}<0$, and if $\sigma_{i 0}<0$, then $\dot{\sigma}_{i 0}>0$. Obviously, we conclude that $\lim _{t \rightarrow \infty} \sigma_{i 0}=0$ and $\lim _{t \rightarrow \infty} \dot{\sigma}_{i 0}=0$; that is, $\lim _{t \rightarrow \infty} \sigma_{i}=\sigma_{0}$ and $\lim _{t \rightarrow \infty} \omega_{i}=0$. 
3.2. Dynamic Tracking Case. The states of the leader are assumed to be dynamic, which can be generated by

$$
\begin{gathered}
\dot{v}=S v, \\
\sigma_{0}=F v,
\end{gathered}
$$

where $v \in \mathbb{R}^{3}$ is bounded, $\sigma_{0} \in \mathbb{R}^{3}, S \in \mathbb{R}^{3 \times 3}$, and $F \in \mathbb{R}^{3 \times 3}$.

Assumption 7. The matrix $S$ has no eigenvalues with positive real parts and the eigenvalues with zero real parts are semisimple.

Remark 8. It can be seen that $\dot{v}=S v$ can generate a large class of reference signals, such as step functions of arbitrary magnitudes, ramp functions of arbitrary slopes, or sinusoidal functions of arbitrary amplitudes and initial phases, and therefore $\sigma_{0}$ can represent various reference signals by linearly combining the components of $v$.

We associate a compensator for each follower as follows:

$$
\dot{\theta}_{i}=S \theta_{i}-\mu \sum_{j \in \bar{N}_{i}} a_{i j}\left(\theta_{i}-\theta_{j}\right)=S \theta_{i}-\bar{\theta}_{i}
$$

with $\theta_{0}=v, \bar{\theta}_{i}=\mu \sum_{j \in \bar{N}_{i}} a_{i j}\left(\theta_{i}-\theta_{j}\right)$, where $\mu$ is a positive constant.

Remark 9. Define $\theta=\left[\theta_{1}^{T} \cdots \theta_{n}^{T}\right]^{T}$ and $\varsigma=\theta-1_{n} \otimes v$; then (23) can be written as

$$
\dot{\varsigma}=\left[\left(I_{n} \otimes S\right)-\mu\left(H_{\varrho(t)} \otimes I_{3}\right)\right] \varsigma,
$$

where $H_{\varrho(t)}=\mathscr{L}_{\varrho(t)}+\operatorname{diag}\left\{a_{10}(t), \ldots, a_{n 0}(t)\right\}$. By Lemma 2 of [29], under Assumptions 2 and 7, the origin of the system (24) is exponentially stable; that is, for all $i=1, \ldots, n$, we have

$$
\lim _{t \rightarrow \infty}\left[\theta_{i}(t)-v(t)\right]=0
$$

Also we get that

$$
\begin{aligned}
\lim _{t \rightarrow \infty} \bar{\theta}_{i}(t) & =0, \\
\lim _{t \rightarrow \infty}\left[\dot{\theta}_{i}(t)-\dot{v}(t)\right] & =\lim _{t \rightarrow \infty}\left[S\left(\theta_{i}(t)-v(t)\right)+\bar{\theta}_{i}(t)\right] \\
& =0 .
\end{aligned}
$$

For this reason, we call (23) a distributed observer of the leader state. Moreover, define

$$
\dot{\sigma}_{r i}=F S \theta_{i}-\alpha\left(\sigma_{i}-F \theta_{i}\right),
$$

where $\alpha$ is a positive constant; we get that

$$
\ddot{\sigma}_{r i}=F S \dot{\theta}_{i}-\alpha\left(\dot{\sigma}_{i}-F \dot{\theta}_{i}\right) \text {. }
$$

Then we associate another auxiliary signal for each follower as

$$
\begin{aligned}
\xi_{i} & =\dot{\sigma}_{i}-\dot{\sigma}_{r i}=\dot{\sigma}_{i}-F S \theta_{i}+\alpha\left(\sigma_{i}-F \theta_{i}\right) \\
& =\dot{\sigma}_{i}-F \dot{\theta}_{i}+\alpha\left(\sigma_{i}-F \theta_{i}\right)-F \bar{\theta}_{i}(t),
\end{aligned}
$$

based upon which, the control input can be chosen as

$$
\begin{aligned}
u_{i} & =\omega_{i}^{\times} J_{i} \omega_{i} \\
& +\frac{J_{i} G^{T}\left(\sigma_{i}\right)}{p_{i}}\left[-\dot{G}\left(\sigma_{i}\right) \omega_{i}-\alpha \dot{\sigma}_{i}+(F S+\alpha F) \dot{\theta}_{i}-\xi_{i}\right] .
\end{aligned}
$$

Theorem 10. Under Assumptions 2 and 7, the leader-following dynamic consensus of system (1) is achieved with the control protocol (31).

Proof. Define a Lyapunov function candidate as

$$
V=\frac{1}{2} \sum_{i=1}^{n} \xi_{i}^{\mathrm{T}} \xi_{i}
$$

With (30), taking the derivative of $V$ gives

$$
\dot{V}=\sum_{i=1}^{n} \xi_{i}^{T} \dot{\xi}_{i}=\sum_{i=1}^{n} \xi_{i}^{T}\left[\ddot{\sigma}_{i}-F S \dot{\theta}_{i}+\alpha\left(\dot{\sigma}_{i}-F \dot{\theta}_{i}\right)\right],
$$

where we have used (23). According to (1) and (31), $\dot{V}$ can be written as

$$
\begin{aligned}
\dot{V} & =\sum_{i=1}^{n} \xi_{i}^{T}\left[G\left(\sigma_{i}\right) \dot{\omega}_{i}+\dot{G}\left(\sigma_{i}\right) \omega_{i}-F S \dot{\theta}_{i}\right. \\
& \left.+\alpha\left(\dot{\sigma}_{i}-F \dot{\theta}_{i}\right)\right]=\sum_{i=1}^{n} \xi_{i}^{T}\left[G\left(\sigma_{i}\right) J_{i}^{-1}\left(-\omega_{i}^{\times} J_{i} \omega_{i}+u_{i}\right)\right. \\
& \left.+\dot{G}\left(\sigma_{i}\right) \omega_{i}-F S \dot{\theta}_{i}+\alpha\left(\dot{\sigma}_{i}-F \dot{\theta}_{i}\right)\right]=-\sum_{i=1}^{n} \xi_{i}^{T} \xi_{i} \leq 0 .
\end{aligned}
$$

Though the network is switching, $V$ is positive in $\xi_{i}$ for all $\sigma_{i}$; that is, $V$ is lower bounded for all $t \geq 0$, and therefore $\xi_{i}$ is bounded. Next we will prove $\lim _{t \rightarrow \infty} \xi_{i}=0$.

As (30) can be written as

$$
\dot{\sigma}_{i}-F \dot{\theta}_{i}+\alpha\left(\sigma_{i}-F \theta_{i}\right)=\xi_{i}-F \bar{\theta}_{i}(t),
$$

then (35) can be viewed as a stable first-order linear system in $\left(\sigma_{i}-F \theta_{i}\right)$ with input $\left(\xi_{i}-F \bar{\theta}_{i}\right)$. Since $\xi_{i}$ is bounded and $\lim _{t \rightarrow \infty} \bar{\theta}_{i}(t)=0$, we get that $\left(\sigma_{i}-F \theta_{i}\right)$ and $\left(\dot{\sigma}_{i}-F \dot{\theta}_{i}\right)$ are both bounded. As $v$ and $\dot{v}$ are bounded, $\theta_{i}$ and $\dot{\theta}_{i}$ are both bounded by (25) and (27). Equations (28) and (29) show the boundedness of $\dot{\sigma}_{r i}$ and $\ddot{\sigma}_{r i}$.

By (30) and $\dot{\xi}_{i}=-\xi_{i}, \xi_{i}$ is continuous and $\dot{\xi}_{i}$ is bounded, together with $\ddot{V}=\sum_{i=1}^{n} \xi_{i}^{T} \dot{\xi}_{i}$; we get that there exists a real number $\gamma>0$ such that

$$
\sup _{t_{i} \leq t \leq t_{i+1}, i=0,1,2, \ldots}|\ddot{V}(t)| \leq \gamma .
$$

Therefore, by invoking Lemma $4, \lim _{t \rightarrow \infty} \dot{V}(t)=0$, and thus $\lim _{t \rightarrow \infty} \xi_{i}=0$.

Since both $\xi_{i}$ and $\bar{\theta}_{i}$ tend to zero as $t \rightarrow \infty$, we get that $\sigma_{i}-F \theta_{i} \rightarrow 0$ and $\dot{\sigma}_{i}-F \dot{\theta}_{i} \rightarrow 0$ as $t \rightarrow \infty$. Therefore, by the following equations

$$
\begin{aligned}
& \sigma_{i}(t)-\sigma_{0}(t)=\left(\sigma_{i}(t)-F \theta_{i}(t)\right)+F\left(\theta_{i}(t)-v(t)\right), \\
& \dot{\sigma}_{i}(t)-\dot{\sigma}_{0}(t)=\left(\dot{\sigma}_{i}(t)-F \dot{\theta}_{i}(t)\right)+F\left(\dot{\theta}_{i}(t)-\dot{v}(t)\right)
\end{aligned}
$$


as well as (25) and (27), we can conclude that $\sigma_{i}-\sigma_{0} \rightarrow 0$ and $\dot{\sigma}_{i}-\dot{\sigma}_{0} \rightarrow 0$ as $t \rightarrow \infty$ for all $i=1, \ldots, n$; that is, $\sigma_{i}-\sigma_{0} \rightarrow 0$ and $\omega_{i}-\omega_{0} \rightarrow 0$.

Remark 11. For uniformly connected switching topologies, each agent keeps connected all the time such that there are no isolated agents, but this does not hold for jointly connected switching topologies. In order to prevent the divergence of the isolated agents, we design different control laws for the connected and isolated agents, respectively. References [20, 21] also study the attitude consensus control problems in the presence of jointly connected switching topologies. However, there is no leader in the system, and the control schemes are not used in the leader-following problem. Meanwhile, the problem in this paper is more difficult as only a subset of followers can access the state information of the leader.

\section{Numerical Example}

In this section, we present two numerical examples to illustrate the effectiveness of our protocols (9) and (31). Consider the attitude consensus problem for five spacecrafts with one single leader and four followers. The dynamic equation of each spacecraft is described as

$$
\begin{aligned}
& \dot{\sigma}_{i}=G\left(\sigma_{i}\right) \omega_{i}, \\
& \dot{\omega}_{i}=J_{i}^{-1}\left(-\omega_{i}^{\times} J_{i} \omega_{i}+u_{i}\right),
\end{aligned}
$$

where $i=1,2,3,4, J_{1}=\cdots=J_{4}=\left[\begin{array}{ccc}10 & 0 & 0 \\ 0 & 20 & 0 \\ 0 & 0 & 10\end{array}\right]$.

The switching topologies $\overline{\mathscr{G}}_{\varrho(t)}$ associated with the system are shown in Figure 1, and the switching sequence is $\overline{\mathscr{G}}_{1} \rightarrow$ $\overline{\mathscr{G}}_{2} \rightarrow \overline{\mathscr{G}}_{3} \rightarrow \overline{\mathscr{G}}_{4} \rightarrow \overline{\mathscr{G}}_{1} \rightarrow \cdots$, which is dictated by the following switching signal:

$$
\varrho(t)= \begin{cases}1, & m \cdot T \leq t<(m+1) T \\ 2, & (m+1) T \leq t<(m+2) T \\ 3, & (m+2) T \leq t<(m+3) T \\ 4, & (m+3) T \leq t<(m+4) T\end{cases}
$$

where $T=1 \mathrm{~s}, m=0,1,2, \ldots$.

In our simulation, we choose $a_{i j}=1, i=1,2,3,4$, if spacecraft $j$ is a neighbor of spacecraft $i$ and $a_{i j}=0$ otherwise.

For the first example, we set the initial attitude and the angular velocity of the leader to be, respectively, $\sigma_{0}=$ $[2,5,6]^{T}$ and $\omega_{0}=[0,0,0]^{T}$. The initial attitudes of the three followers are set to be, respectively, $\sigma_{1}=[1,6,3]^{T}, \sigma_{2}=$ $[5,4,1]^{T}, \sigma_{3}=[4,1,6]^{T}$, and $\sigma_{4}=[1,5,2]^{T}$, and the initial angular velocities of the three followers are set to be, respectively, $\omega_{1}=[2,3,2]^{T}, \omega_{2}=[4,3,2]^{T}, \omega_{3}=[1,4,2]^{T}$, and $\omega_{4}=[3,2,4]^{T}$. The control parameters are chosen as $c=2$ and $\gamma=2$. The attitude errors between the four followers and the leader are shown in Figure 2, and the angular velocities are shown in Figure 3. Besides, Figure 4 shows the auxiliary vector errors. We see that the regulation control goal is achieved under the jointly connected topologies.

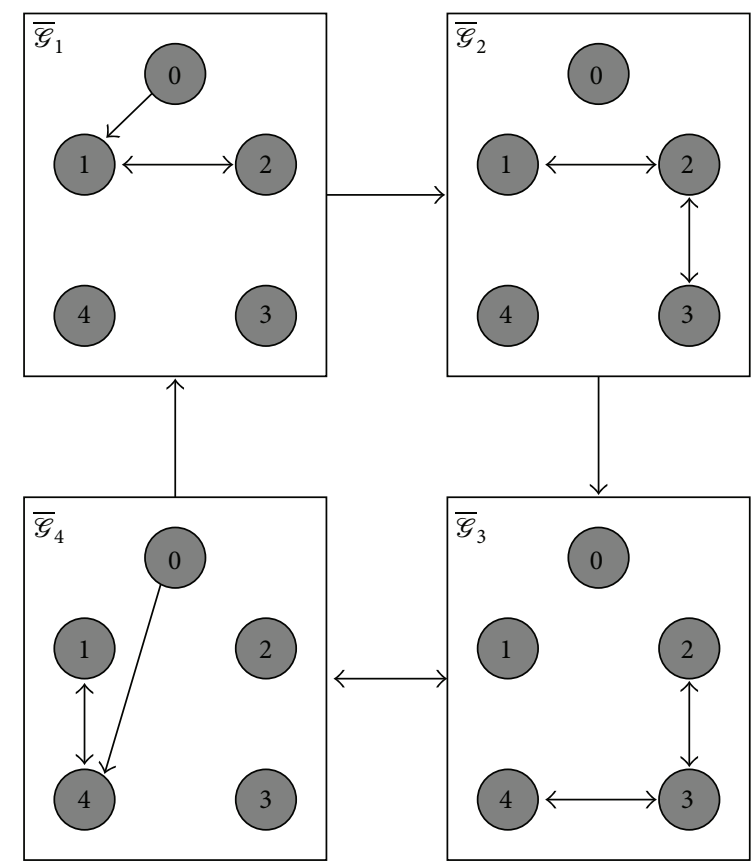

FIGURE 1: The switching network topologies.

For the second example, the reference signal is set to be

$$
\begin{aligned}
& \sigma_{01}(t)=-\frac{\pi}{6} \sin \left(\frac{\pi}{10} t\right)-\frac{\pi}{3} \\
& \sigma_{02}(t)=-\frac{\pi}{3} \sin \left(\frac{\pi}{10} t\right)+\frac{\pi}{6} \\
& \sigma_{03}(t)=-\frac{\pi}{6} \cos \left(\frac{\pi}{10} t\right)+\frac{\pi}{2}
\end{aligned}
$$

which can be generated by a leader system of the form (22) with

$$
\begin{aligned}
& S=\left[\begin{array}{ccc}
0 & \frac{\pi}{10} & 0 \\
-\frac{\pi}{10} & 0 & 0 \\
0 & 0 & 0
\end{array}\right], \\
& F=\left[\begin{array}{ccc}
0 & \frac{\pi}{6} & -\frac{\pi}{3} \\
0 & \frac{\pi}{3} & \frac{\pi}{6} \\
\frac{\pi}{6} & 0 & \frac{\pi}{2}
\end{array}\right],
\end{aligned}
$$

and $v(0)=[-1,0,1]^{T}$. The initial attitudes of the three followers are set to be, respectively, $\sigma_{1}=[1.1,1.5,-0.2]^{T}$, $\sigma_{2}=[1.5,1.1,0.1]^{T}, \quad \sigma_{3}=[0.1,-0.4,1.6]^{T}$, and $\sigma_{4}=$ $[-0.4,0.1,1.3]^{T}$, and the initial angular velocities of the three followers are set to be, respectively, $\omega_{1}=[0.2,0.3,0.2]^{T}, \omega_{2}=$ $[0.4,0.3,0.2]^{T}, \omega_{3}=[0.1,0.4,0.2]^{T}$, and $\omega_{4}=[0.3,0.2,0.4]^{T}$. The control parameters are chosen as $\alpha=2$ and $\mu=5$. The attitude errors between the four followers and the leader are 

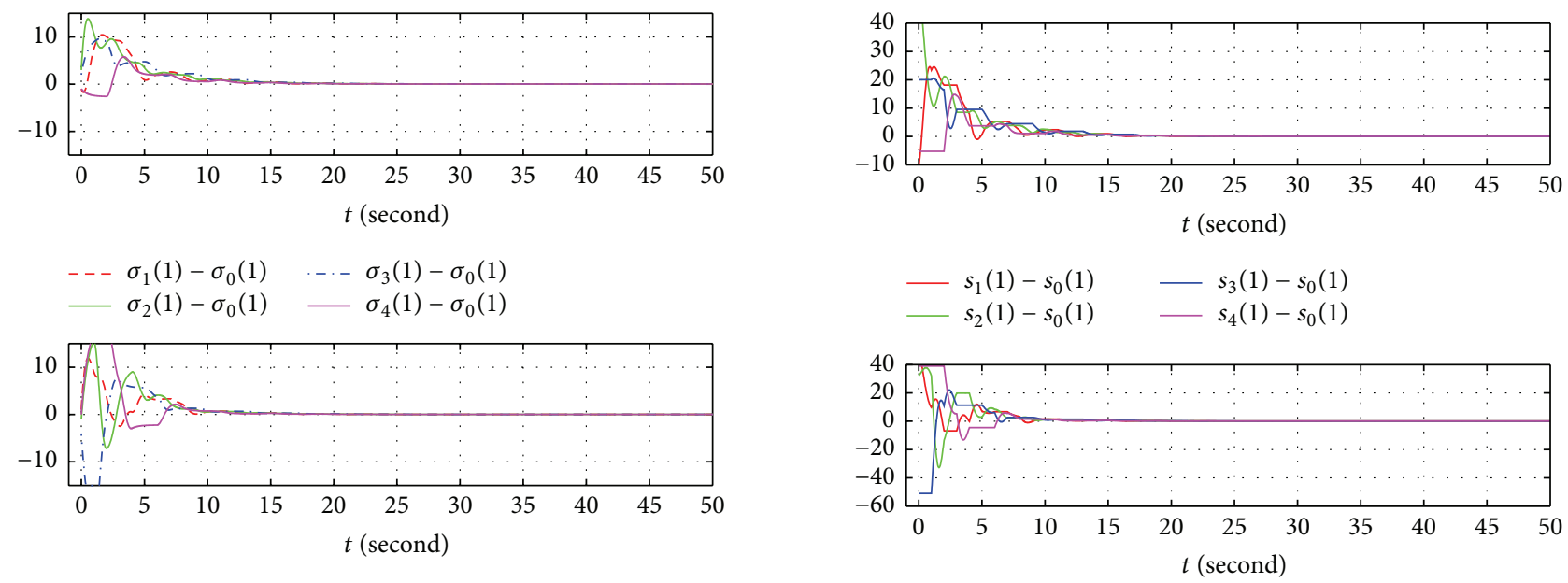

$$
\begin{array}{rr}
-\sigma_{1}(2)-\sigma_{0}(2) \quad--\sigma_{3}(2)-\sigma_{0}(2) \\
-\sigma_{2}(2)-\sigma_{0}(2) \quad-\sigma_{4}(2)-\sigma_{0}(2)
\end{array}
$$

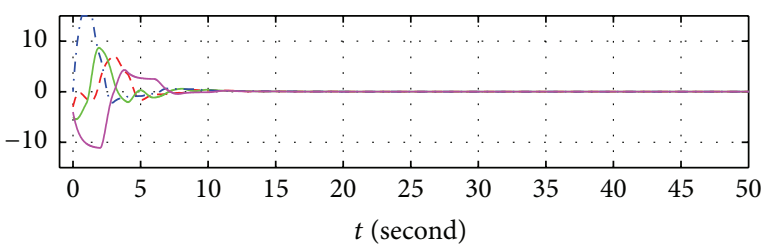

$$
\begin{aligned}
& -s_{1}(2)-s_{0}(2) \quad-s_{3}(2)-s_{0}(2) \\
& -s_{2}(2)-s_{0}(2) \quad-s_{4}(2)-s_{0}(2)
\end{aligned}
$$

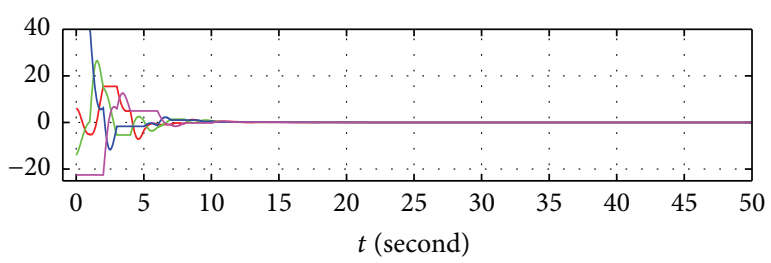

$$
\begin{array}{r}
-\sigma_{1}(3)-\sigma_{0}(3) \quad--\sigma_{3}(3)-\sigma_{0}(3) \\
-\sigma_{2}(3)-\sigma_{0}(3) \quad-\sigma_{4}(3)-\sigma_{0}(3)
\end{array}
$$

FIgUre 2: The attitude error $\sigma_{i}-\sigma_{0}$.

$$
\begin{aligned}
& -s_{1}(3)-s_{0}(3) \quad-s_{3}(3)-s_{0}(3) \\
& -s_{2}(3)-s_{0}(3) \quad-s_{4}(3)-s_{0}(3)
\end{aligned}
$$

FIgURE 4: The auxiliary vector error $s_{i}-s_{0}$.
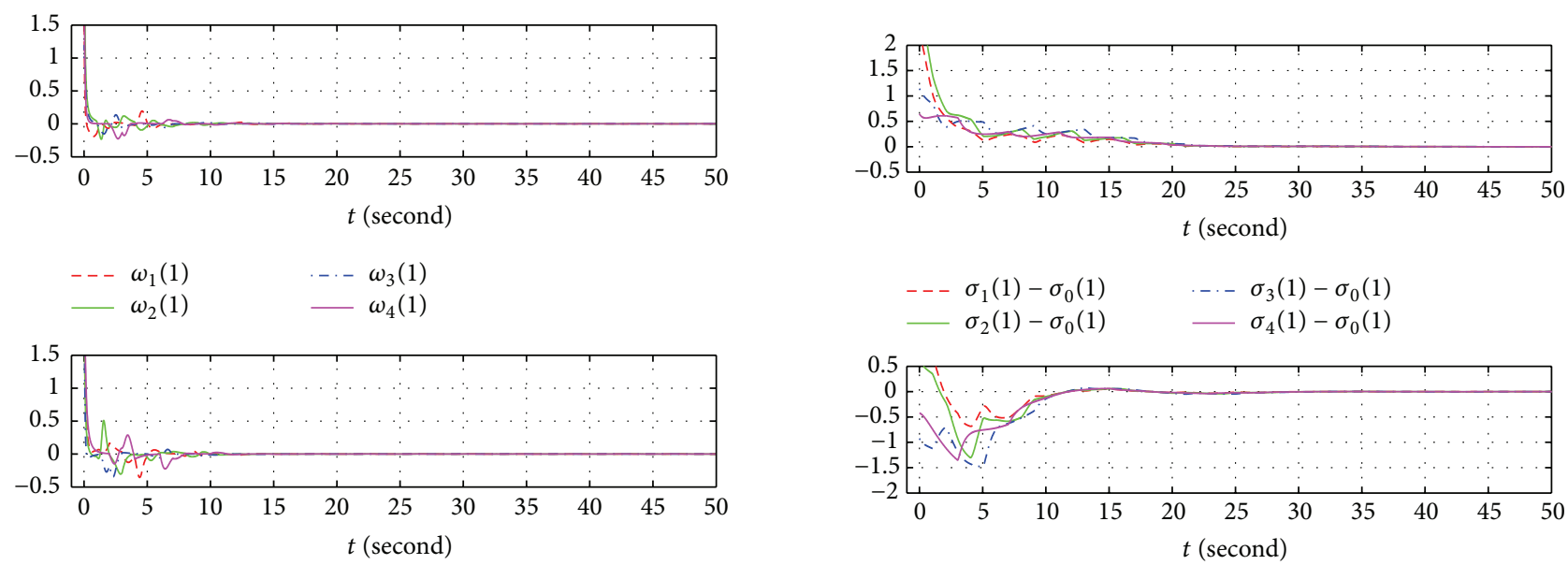

$$
\begin{array}{rr}
--\sigma_{1}(1)-\sigma_{0}(1) & -\cdot-\sigma_{3}(1)-\sigma_{0}(1) \\
-\sigma_{2}(1)-\sigma_{0}(1) & -\sigma_{4}(1)-\sigma_{0}(1)
\end{array}
$$

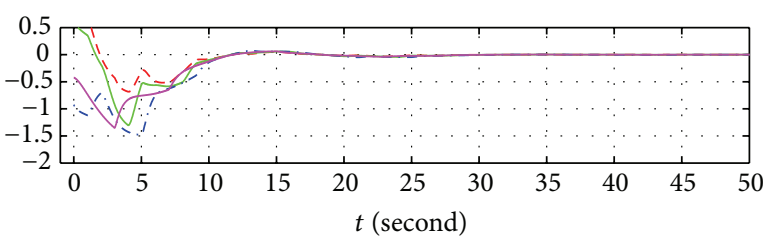

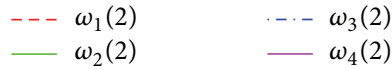

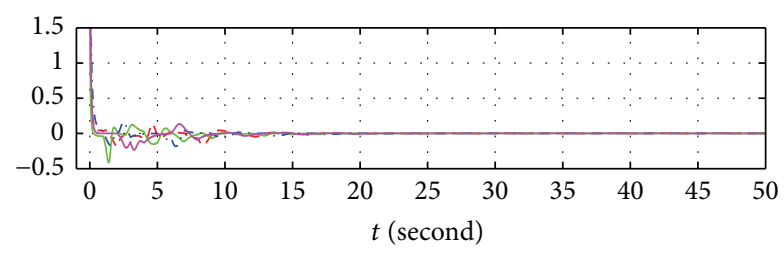

$$
\begin{aligned}
& --\sigma_{1}(2)-\sigma_{0}(2) \quad \cdots-\sigma_{3}(2)-\sigma_{0}(2) \\
& -\sigma_{2}(2)-\sigma_{0}(2) \quad-\sigma_{4}(2)-\sigma_{0}(2)
\end{aligned}
$$

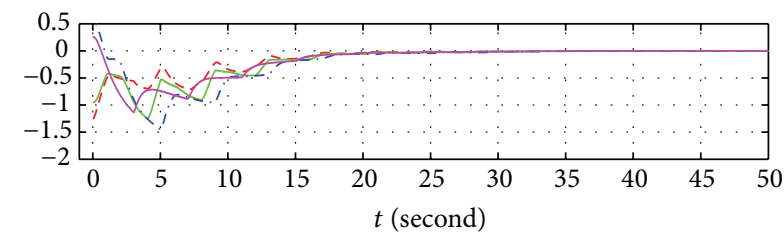

$$
\begin{array}{rr}
-\omega_{1}(3) & -\cdot \omega_{3}(3) \\
- & \omega_{2}(3)
\end{array}
$$

FIgURE 3: The angular velocity $\omega_{i}$.

$-\sigma_{1}(3)-\sigma_{0}(3) \quad \ldots \sigma_{3}(3)-\sigma_{0}(3)$
$-\sigma_{2}(3)-\sigma_{0}(3) \quad-\sigma_{4}(3)-\sigma_{0}(3)$

Figure 5: The attitude error $\sigma_{i}-\sigma_{0}$. 

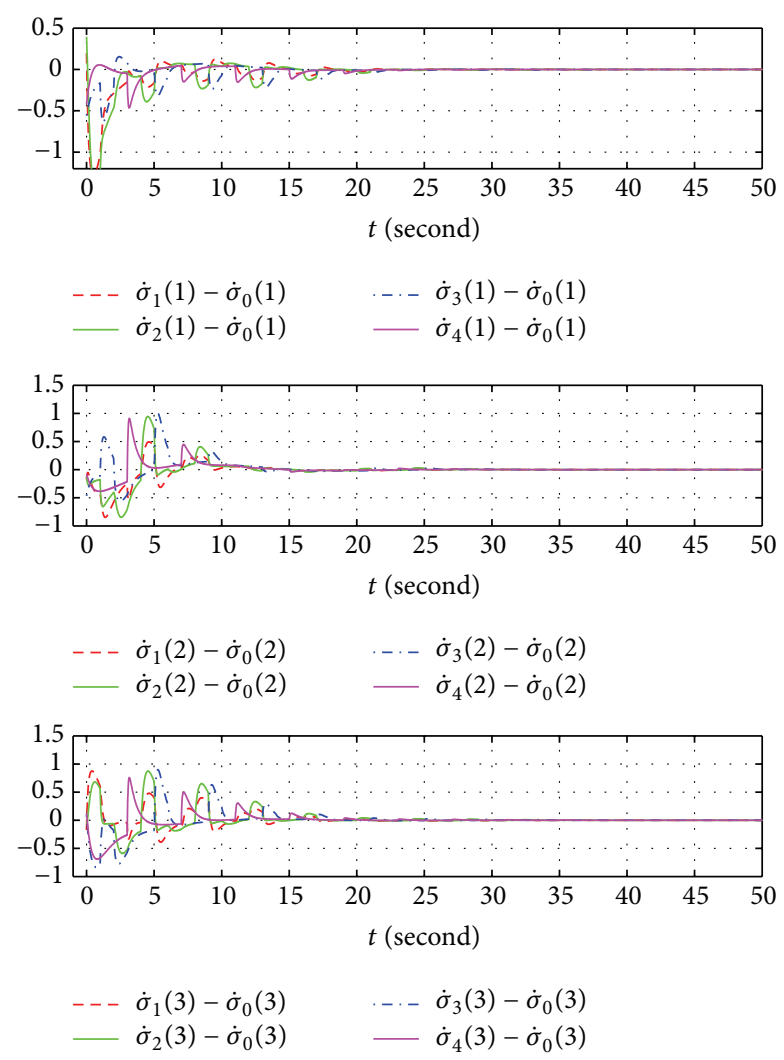

Figure 6: The angular velocity error $\dot{\sigma}_{i}-\dot{\sigma}_{0}$.
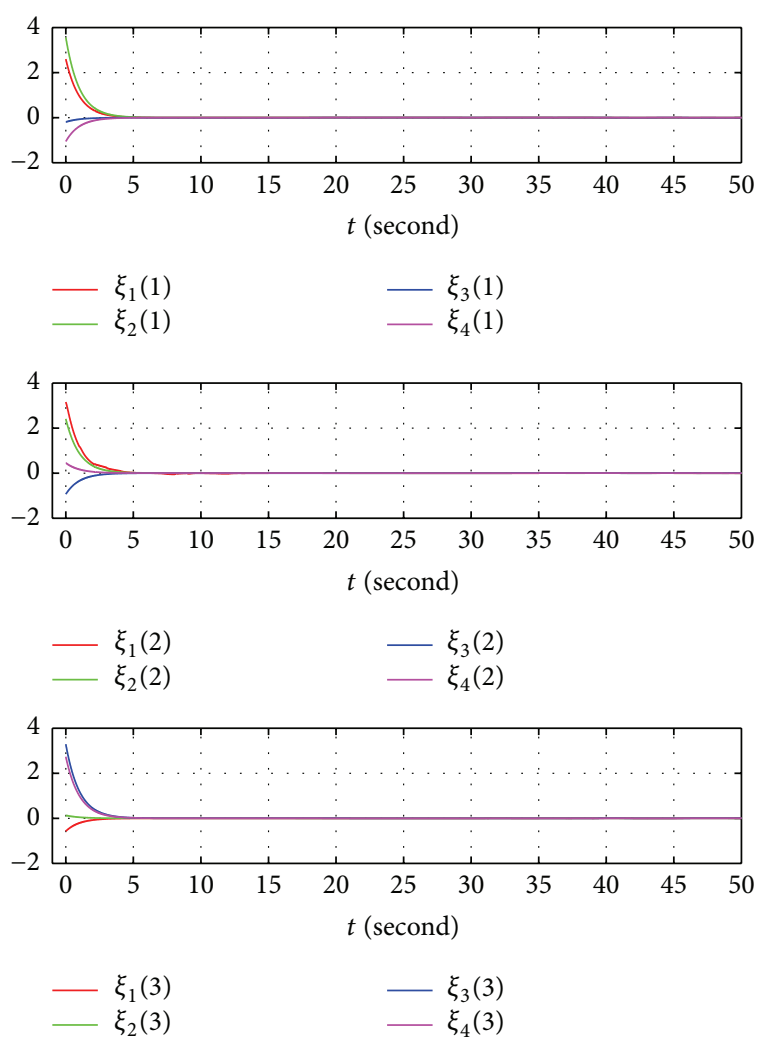

FIgURE 7: The auxiliary vectors $\xi_{i}$. shown in Figure 5, and the angular velocities are shown in Figure 6. Besides, Figure 7 shows the auxiliary vectors. We see that the dynamic tracking control goal is achieved under the jointly connected topologies.

\section{Conclusion}

In this paper, the leader-following attitude consensus problem with a single leader under jointly connected topologies is studied for two different cases, that is, the regulation case with a static leader and the dynamic tracking case with a dynamic leader. Auxiliary vectors are constructed, based upon which the control protocols are designed for each follower spacecraft. Numerical simulation results are presented to illustrate the effectiveness of the controllers. In the future, we will study the problem under jointly connected topologies coupled with time delays.

\section{Competing Interests}

The authors declare that they have no competing interests.

\section{References}

[1] D. Folta, J. Bristow, A. Hawkins, and G. Dell, "Nasa’s autonomous formation flying technology demonstration, earthobserving-1," in Proceedings of the 1st International Symposium on Formation Flying Missions and Technologies, Centre National d'Etudes Spa-Tiales, pp. 71-76, Toulouse, France, October 2002.

[2] S. P. Neeck, T. J. Magner, and G. E. Paules, "NASA's small satellite missions for Earth observation," Acta Astronautica, vol. 56, no. 1-2, pp. 187-192, 2005.

[3] P. Lawson, "The terrestrial planet finder," in Proceedings of the IEEE Aerospace Conference, pp. 2005-2011, Big Sky, Mont, USA, March 2001.

[4] W. Kang and H.-H. Yeh, "Coordinated attitude control of multisatellite systems," International Journal of Robust and Nonlinear Control, vol. 12, no. 2-3, pp. 185-205, 2002.

[5] D. P. Scharf, F. Y. Hadaegh, and S. R. Ploen, "A survey of spacecraft formation flying guidance and control (Part II): control," in Proceedings of the 2004 American Control Conference (AAC '04), pp. 2976-2985, Boston, Mass, USA, July 2004.

[6] S. M. Joshi, A. G. Kelkar, and J. T. Wen, "Robust attitude stabilization of spacecraft using nonlinear quaternion feedback," IEEE Transactions on Automatic Control, vol. 40, no. 10, pp. 1800-1803, 1995.

[7] J. R. Lawton and R. W. Beard, "Synchronized multiple spacecraft rotations," Automatica, vol. 38, no. 8, pp. 1359-1364, 2002.

[8] R. Kristiansen, A. Loría, A. Chaillet, and P. J. Nicklasson, "Spacecraft relative rotation tracking without angular velocity measurements," Automatica, vol. 45, no. 3, pp. 750-756, 2009.

[9] A. Abdessameud, A. Tayebi, and I. G. Polushin, "Attitude synchronization of multiple rigid bodies with communication delays," IEEE Transactions on Automatic Control, vol. 57, no. 9, pp. 2405-2411, 2012.

[10] J. T. Lyu and D. Gao, "Attitude synchronization for multiple spacecraft with input constraints," Chinese Journal of Aeronautics, vol. 27, no. 2, pp. 321-327, 2014. 
[11] I. Okoloko and Y. Kim, "Attitude synchronization of multiple spacecraft with cone avoidance constraints," in Proceedings of the IEEE Aerospace Conference, pp. 1-10, Big Sky, Mont, USA, March 2012.

[12] L. Ma, S. Wang, H. Min, S. Liao, and Y. Liu, "Distributed finitetime attitude containment control of multi-rigid-body systems," Journal of the Franklin Institute, vol. 352, no. 5, pp. 2187-2203, 2015.

[13] H. Cai and J. Huang, "The leader-following attitude control of multiple rigid spacecraft systems," Automatica, vol. 50, no. 4, pp. 1109-1115, 2014.

[14] A. Abdessameud and A. Tayebi, "Attitude synchronization of a group of spacecraft without velocity measurements," IEEE Transactions on Automatic Control, vol. 54, no. 11, pp. 26422648, 2009.

[15] Z. Y. Meng, Z. You, G. H. Li, and C. S. Fan, "Cooperative attitude control of multiple rigid bodies with multiple time-varying delays and dynamically changing topologies," Mathematical Problems in Engineering, vol. 2010, Article ID 621594, 19 pages, 2010.

[16] E. D. Jin and S. W. Zhaowei, "Robust attitude synchronisation controllers design for spacecraft formation," IET Control Theory \& Applications, vol. 3, no. 3, pp. 325-339, 2009.

[17] P. Bi, J.-J. Luo, and B. Zhang, "Cooperate control algorithm for spacecraft formation flying based on consensus theory," Journal of Astronautics, vol. 31, no. 1, pp. 70-74, 2010.

[18] A. Sarlette, R. Sepulchre, and N. E. Leonard, "Autonomous rigid body attitude synchronization," Automatica, vol. 45, no. 2, pp. 572-577, 2009.

[19] B.-Q. Zhang, S.-M. Song, and X.-L. Chen, "Robust coordinated control for formation flying satellites with time delays and switching topologies," Journal of Astronautics, vol. 33, no. 7, pp. 910-919, 2012.

[20] J. Thunberg, W. J. Song, E. Montijano, Y. G. Hong, and X. M. Hu, "Distributed attitude synchronization control of multiagent systems with switching topologies," Automatica, vol. 50, no. 3, pp. 832-840, 2014.

[21] S. Chen, P. Shi, W. Zhang, and L. Zhao, "Finite-time consensus on strongly convex balls of Riemannian manifolds with switching directed communication topologies," Journal of Mathematical Analysis and Applications, vol. 409, no. 2, pp. 663675, 2014.

[22] M. D. Shuster, "A survey of attitude representations," Journal of Astronautical Sciences, vol. 41, no. 4, pp. 493-517, 1993.

[23] P. Tsiotras, "Further passivity results for the attitude control problem," IEEE Transactions on Automatic Control, vol. 43, no. 11, pp. 1597-1600, 1998.

[24] H. Du, S. Li, and C. Qian, "Finite-time attitude tracking control of spacecraft with application to attitude synchronization," IEEE Transactions on Automatic Control, vol. 56, no. 11, pp. 2711-2717, 2011.

[25] P. Tsiotras, "Stabilization and optimality results for the attitude control problem," Journal of Guidance, Control, and Dynamics, vol. 19, no. 4, pp. 772-779, 1996.

[26] W. Ni and D. Cheng, "Leader-following consensus of multiagent systems under fixed and switching topologies," Systems \& Control Letters, vol. 59, no. 3-4, pp. 209-217, 2010.

[27] J. J. E. Slotine and W. P. Li, Applied Nonlinear Control, Prentice Hall, Upper Saddle River, NJ, USA, 1991.

[28] Y. Su and J. Huang, "Stability of a class of linear switching systems with applications to two consensus problems," IEEE
Transactions on Automatic Control, vol. 57, no. 6, pp. 1420-1430, 2012.

[29] Y. Su and J. Huang, "Cooperative output regulation with application to multi-agent consensus under switching network," IEEE Transactions on Systems, Man, and Cybernetics, Part B: Cybernetics, vol. 42, no. 3, pp. 864-875, 2012. 


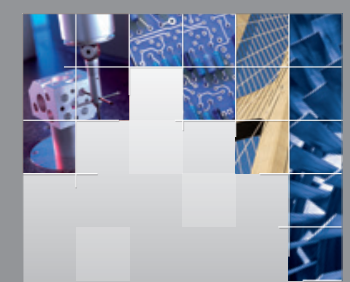

\section{Enfincering}
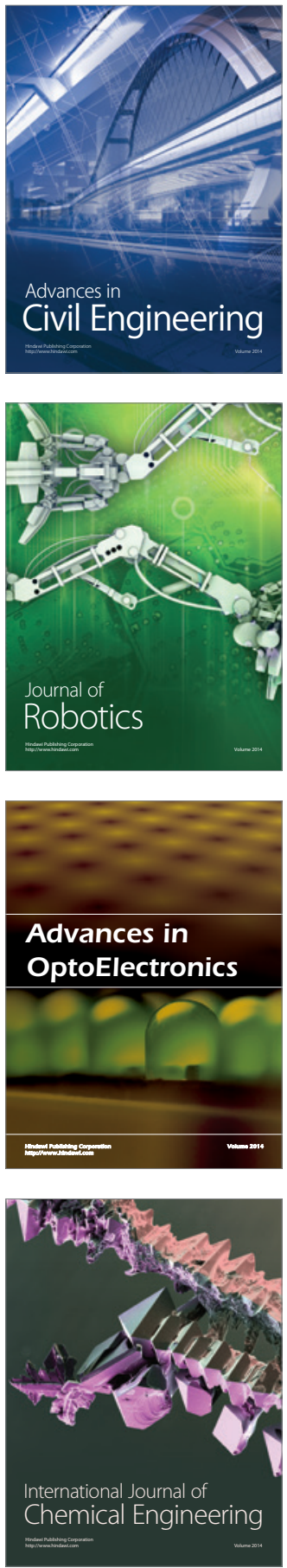

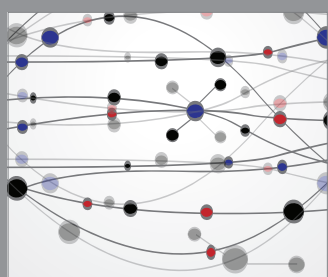

The Scientific World Journal

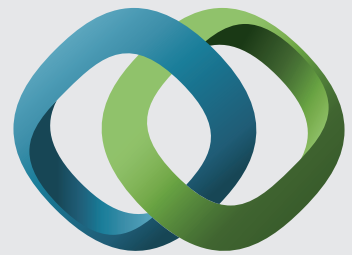

\section{Hindawi}

Submit your manuscripts at

http://www.hindawi.com
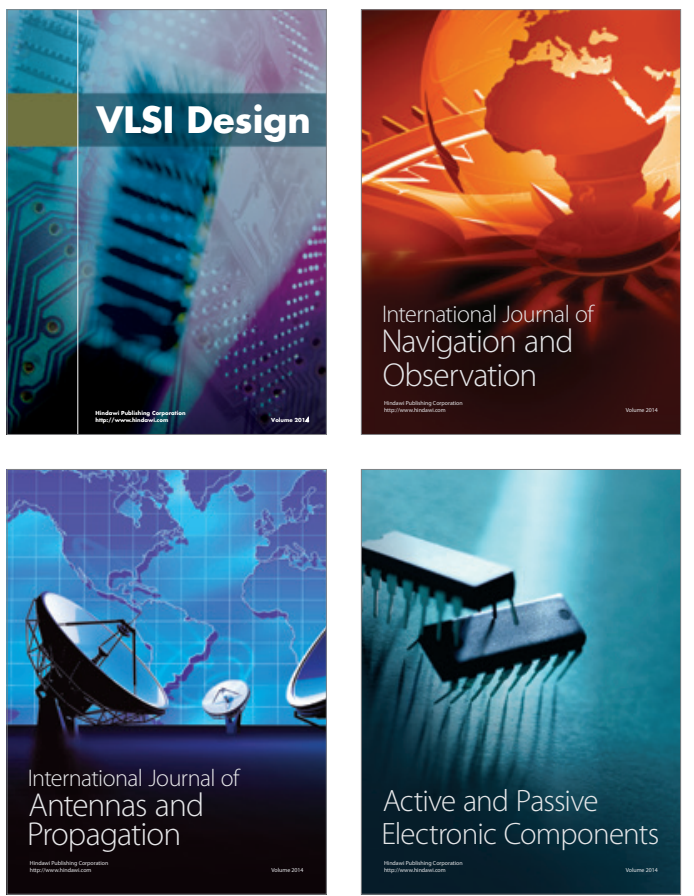
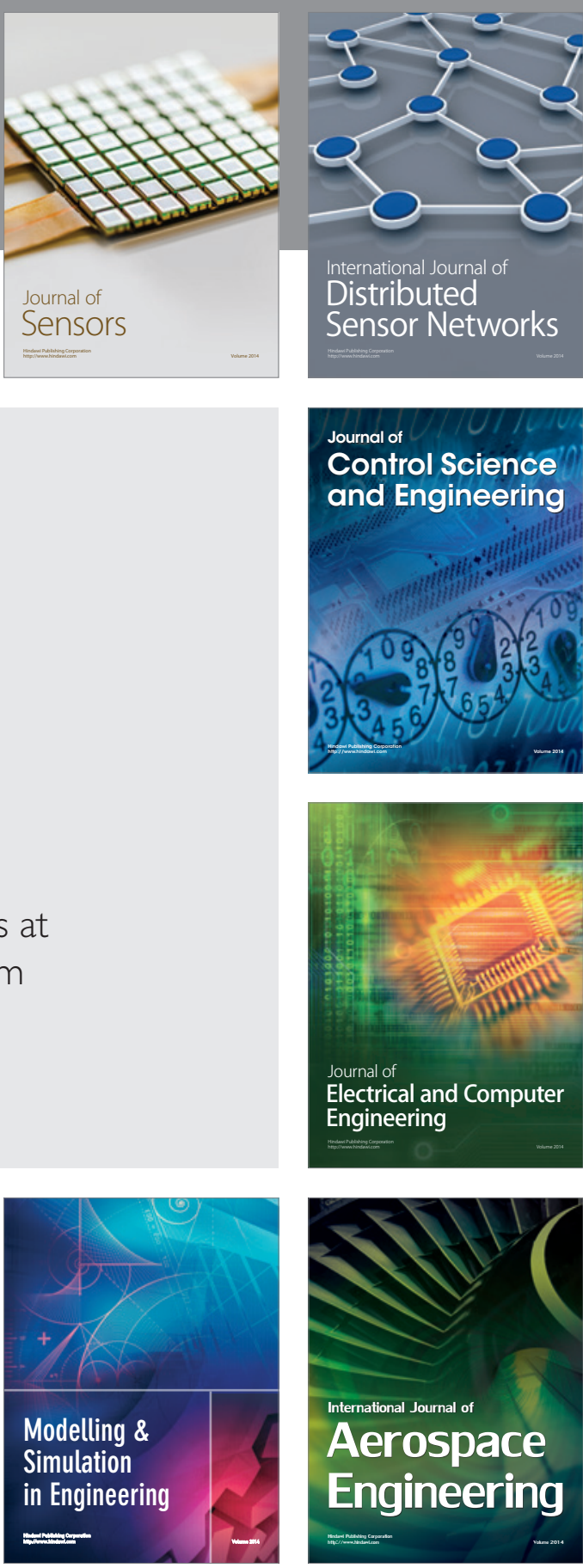

International Journal of

Distributed

Sensor Networks

Journal of

Control Science

and Engineering
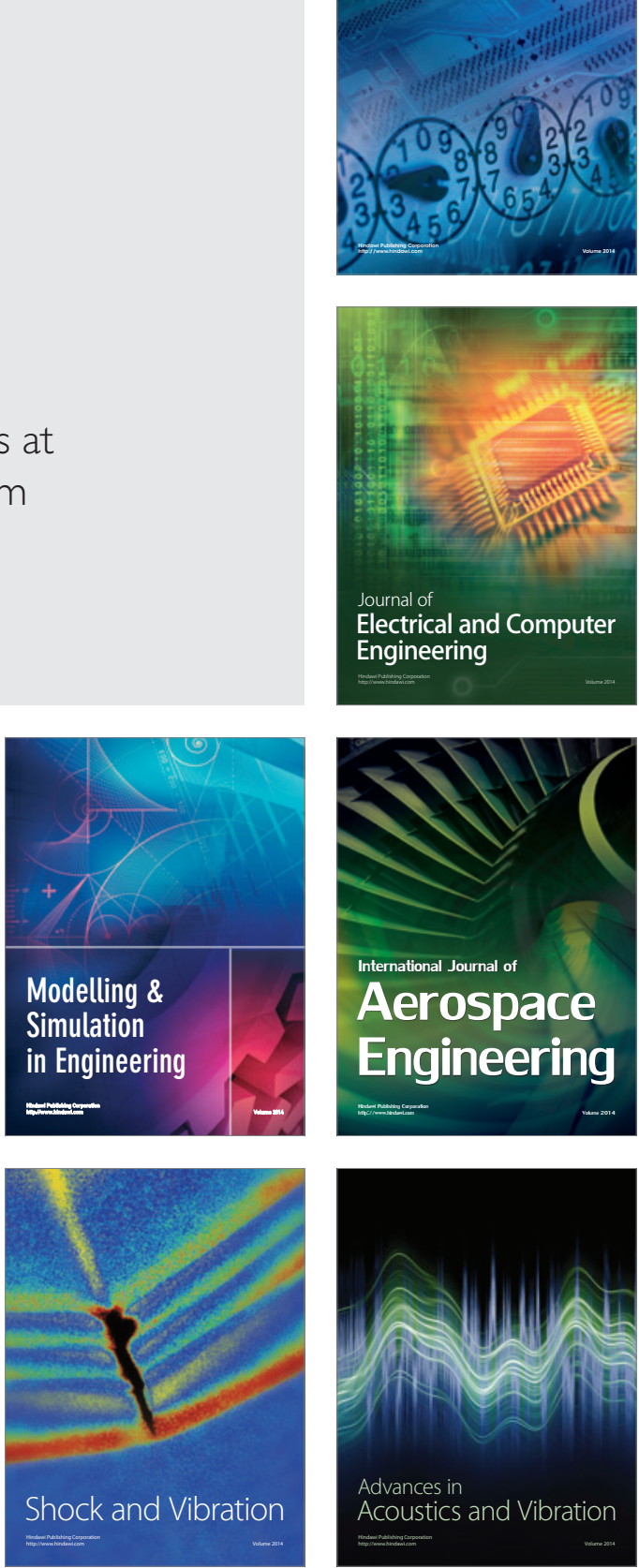